\title{
Les bus touristiques, une technologie spatiale pour habiter les métropoles. Le cas de Los Angeles
}

Tourist buses: a spatial technology to inhabit metropolises. The case of Los

Angeles

\section{Léopold Lucas}

\section{OpenEdition}

\section{Journals}

Édition électronique

URL : http://journals.openedition.org/tourisme/1671

DOI : 10.4000/tourisme.1671

ISSN : 2492-7503

\section{Éditeur}

Éditions touristiques européennes

\section{Référence électronique}

Léopold Lucas, «Les bus touristiques, une technologie spatiale pour habiter les métropoles. Le cas de Los Angeles », Mondes du Tourisme [En ligne], 14 | 2018, mis en ligne le 30 juin 2018, consulté le 30 avril 2019. URL : http://journals.openedition.org/tourisme/1671 ; DOI : 10.4000/tourisme.1671

Ce document a été généré automatiquement le 30 avril 2019.

\section{(i) $(8)$}

Mondes du tourisme est mis à disposition selon les termes de la licence Creative Commons Attribution - Pas d'Utilisation Commerciale - Pas de Modification 4.0 International. 


\section{Les bus touristiques, une technologie spatiale pour habiter les métropoles. Le cas de Los Angeles}

Tourist buses: a spatial technology to inhabit metropolises. The case of Los Angeles

Léopold Lucas

\section{Introduction}

1 Malgré l'importance et la vaste diffusion à l'échelle mondiale des services de bus touristiques, peu de recherches se sont penchées sur ce phénomène; celles qui l'ont fait se sont focalisées en particulier aux promoteurs, au tracé des parcours et aux effets sur l'espace urbain (Cunin et Rinaudo, 2008; Daniels et al., 2018; Farias, 2010a, 2010b; Lumsdon, 2008). De façon complémentaire à ces travaux, l'objectif de cet article est de s'intéresser aux usages et pratiques de ce service par les touristes. Plutôt que de le considérer de façon trop simpliste, et selon la connotation négative classique (qui apparait, on le verra, dans les propos de certains touristes), comme un symbole du «tourisme de masse », la proposition de cet article consiste en effet à envisager les bus touristiques comme une véritable technologie spatiale (Équipe MIT, 2002) qui aide les individus à gérer cet «horizon d'altérité» (Lazzarotti, 2006) que constitue le lieu touristique. Cela constitue ainsi une entrée fondamentale pour la compréhension des manières dont les touristes font avec l'espace: les individus et leurs pratiques sont au cœur de ce système. Cela permet d'investir autrement la question de recherche suivante : dans quelle mesure les individus sont-ils capables d'habiter touristiquement une métropole?

2 Cette analyse fait suite à une réflexion sur les parcours des touristes qui n'utilisent pas les bus touristiques à Los Angeles (Lucas, 2018) : l'enquête montre une grande différence, notamment dans l'ampleur des cheminements, selon la maittrise de techniques dont 
dispose les individus. Il s'agit ici de compléter l'investigation en envisageant l'autre face de ce problème, en se focalisant spécifiquement sur les arbitrages des touristes qui font appel à un service proposant un itinéraire touristique défini. Le point de départ de la réflexion consiste à insister sur l'idée qu'habiter - entendu ici dans son acception désignant le «faire avec de l'espace » (Stock, 2012) - ne va pas de soi, n'est pas évident. $\mathrm{Au}$ contraire, la pratique d'un lieu, et à plus forte raison s'il n'est pas familier et s'il possède un degré d'altérité plus ou moins important (ce qui est le cas en situation touristique), est problématique. L'une des perspectives théoriques défendue ici consiste à envisager toute configuration urbaine comme un enchevêtrement d'épreuves spatiales: habiter consiste alors à "faire avec» différentes épreuves spatiales. Ce cadre théorique s'appuie sur la proposition de Michel Lussault (2013) de dégager six compétences spatiales élémentaires. L'hypothèse soutenue ici est que ces compétences ne sont $\mathrm{ni}$ élémentaires ni spécifiquement spatiales (tout du moins qu'il est possible d'envisager d'autres types de compétences pouvant intervenir dans les manières de faire avec de l'espace). La perspective adoptée suggère plutôt de partir des problèmes auxquels les compétences sont supposées apporter des solutions, pour essayer de comprendre comment les individus résolvent (ou non) ces problèmes, quelles compétences ils mobilisent (ou non). La proposition est donc de dégager des épreuves spatiales. De façon synthétique, il est possible d'en dégager au moins trois grandes familles : i) placement, ii) distances, iii) limites. Ces épreuves sont de nature et d'intensité variables, d'une part selon la configuration spatiale, d'autre part en fonction de la personne qui y est confrontée. Chaque espace, quelle que soit son échelle (d'une pièce au monde), implique ce genre d'épreuves : tout individu se trouve constamment dans l'obligation de répondre à ces enjeux. Pour gérer ces épreuves, la posture défendue est que les individus doivent mobiliser i) un "stock de connaissances" (Schutz, 1987), que l'on peut définir synthétiquement comme l'ensemble des informations dont dispose un individu, et ii) des compétences - que l'on entend ici, suivant une expression de Wittgenstein (1984), comme la maîtrise de techniques, nécessaire pour littéralement «mettre en pratique » ces connaissances.

3 Le cheminement peut s'intégrer à l'épreuve que constitue la gestion des distances. Mais cela va plus loin que d'aller d'un point $\mathrm{A}$ à un point $\mathrm{B}$ : de façon plus complexe, cela correspond à l'élaboration et à la réalisation d'un itinéraire, c'est-à-dire tout à la fois la mise en relation pratique mais également la mise en sens d'un ensemble de lieux. Établir et réaliser un parcours dans l'espace urbain, cheminer entre les différents lieux touristiques d'une agglomération est un défi majeur. C'est précisément cet aspect qui est au cœur du système des circuits effectués par les bus touristiques. L'objectif de ce papier est de discuter l'utilisation des bus touristiques à l'aune des compétences/connaissances des individus, en avançant l'hypothèse que ce service, tout en étant une pratique touristique en tant que telle, constitue en même temps une technologie spatiale utilisée par les individus pour faire plus facilement avec l'espace. Le bus n'est pas qu'une attraction, mais un outil permettant non seulement d'apporter une réponse à la gestion des distances mais plus encore à la mise en relation des lieux.

4 L'enjeu du parcours est proéminent à Los Angeles, sans doute plus qu'ailleurs: l'agglomération, qui fut la «première ville américaine» (Weinstein, 1996), s'est progressivement développée à partir d'une urbanité à la fois privée d'espace public (Ghorra-Gobin, 1997) et de métriques pédestres (Lévy, 1999), la plage étant l'un des rares espaces communs de cette agglomération (Augustin, 2001). De façon synthétique, la 
configuration urbaine de Los Angeles se caractérise ainsi tout à la fois par i) un étalement urbain considérable, ii) une urbanisation qui ne s'est pas constituée autour d'un centre et iii) la prégnance de la métrique automobile ${ }^{1}$. Plus encore, le Central Tourists District (Duhamel et Knafou, 2007), c'est-à-dire les lieux où l'on peut constater «la présence visible et importante des touristes", qui correspond au centre-ville pour les autres métropoles, s'étale ici sur une trentaine de kilomètres (Fainstein et Gladstone, 2001; Lucas, 2014). Ces caractéristiques impliquent deux conséquences majeures pour les touristes quant à la manière d'habiter cette métropole : l'espace touristique est très étalé et la métrique pédestre n'est pas pertinente pour se déplacer d'un lieu à un autre. Au regard de ces spécificités, le concept de Métapole touristique a été proposé pour qualifier cette aire urbaine en tant que lieu touristique (Lucas, 2011).

5 Afin d'investir empiriquement cette question, cet article analyse, après une rapide description du système dans le contexte angeleno, la manière dont cette technologie spatiale est intégrée dans les arbitrages effectués par les touristes concernant la gestion des distances et l'établissement d'un parcours pour découvrir la métropole. L'argumentation s'appuie sur une connaissance pratique de Los Angeles, nourrie par une expérience de cinq terrains d'un mois, effectués à différentes saisons (Lucas, 2014). L'entretien avec des touristes a constitué la technique au cœur du dispositif méthodologique de cette enquête. Ainsi, près de soixante-dix entretiens, de durée et de qualité variables, ont été réalisés avec des touristes; une très large majorité d'entre eux s'est déroulée sur place, notamment au Motel 6 d'Hollywood, qui a été l'un de nos lieux de séjour et dont le hall d'accueil était particulièrement adapté à la conduite des entretiens. Afin de limiter les biais (on peut faire l'hypothèse que les touristes choisissant un même emplacement ont un profil relativement similaire), une autre partie des entretiens a été effectuée dans d'autres contextes, par exemple sur la passerelle qui offre un point de vue sur le Hollywood Sign ou en abordant directement les individus dans certains lieux touristiques. Quelques entretiens ont été réalisés dans d'autres situations, l'un par exemple à Chicago avec un Australien, et quelques autres là où la recherche était dirigée. Cette recherche sur les pratiques et les discours des touristes s'est appuyée de façon complémentaire sur la prise en compte de commentaires de touristes sur Tripadvisor, afin d'accéder à des discours non sollicités, sans le biais des questions posées par le chercheur : ce qui est posté sur cette plateforme correspond alors à de véritables enjeux pour les utilisateurs, suffisamment importants à leurs yeux pour justifier le temps pris pour l'exprimer. Le critère central dans la constitution de l'échantillon était d'obtenir la diversité la plus large possible non seulement des profils sociologiques (nationalité, âge les touristes interrogés étant finalement tous issus de la classe moyenne) mais également des manières de faire avec l'espace (modes de déplacement, lieux de séjour, etc.). L'intérêt des entretiens était d'appréhender le discours de justification des touristes quant à leurs manières de faire avec l'espace à Los Angeles : il s'agissait de suivre les personnes dans leurs raisonnements en soulevant les contradictions, les paradoxes, et en les mettant en perspective avec les observations réalisées in situ. Il ne s'agissait donc pas d'aboutir à une simple description de leurs séjours et de leurs pratiques, mais plutôt de cerner les stratégies, les tactiques et les arbitrages qu'ils mettent en place pour habiter touristiquement cette métropole, de comprendre les logiques qui présidaient à leurs choix dans la façon de se placer, de se déplacer, de parcourir l'espace urbain. Le guide d'entretien, tout autant que l'analyse de ces entretiens, a consisté à saisir ce que les individus peuvent, mais peut-être plus encore sur ce qu'ils ne peuvent pas faire, 
notamment en identifiant le lexique issu du registre de la capacité ( $«$ je peux/ je ne peux pas »; « je sais/ je ne sais pas »).

6 Plus spécifiquement pour cette problématique des bus touristiques, le dispositif méthodologique a reposé sur deux stratégies d'enquête supplémentaires. La première a consisté à participer à des circuits pour en comprendre le fonctionnement de l'intérieur, relever les routes effectivement empruntées et donc les sélections spatiales établies à travers l'espace urbain, noter les éléments mis en avant dans le discours des opérateurs et saisir les réactions et pratiques des touristes. Cela correspondait pleinement à des sessions d'observation participante. La deuxième stratégie résulte du fait d'avoir réussi à sympathiser avec le propriétaire de l'une de ces petites entreprises de vans touristiques sur Hollywood Boulevard, Joseph. Grâce à une présence légitimée, il a ainsi été possible d'être au cœur de cette pratique en passant de nombreuses heures pleinement immergé sur leur stand pour effectuer des séances d'observation - cette fois-ci afin de comprendre les stratégies des vendeurs, les interactions avec les touristes, les pratiques de ces derniers - mais également en ayant la possibilité d'aborder les touristes qui attendaient leur tour (voir illustration 1). Le raisonnement proposé repose sur toute cette connaissance issue du terrain et surtout sur la restitution des observations: de longs extraits d'entretiens permettant d'avoir une description précise des pratiques par les protagonistes eux-mêmes et la succession de petits extraits montrant la récurrence des arguments justificatifs avancés par les touristes et attestant l'arrivée à un certain degré de saturation des données.

Illustration 1. Stand d'une entreprise de vans touristiques, lieu d'enquête

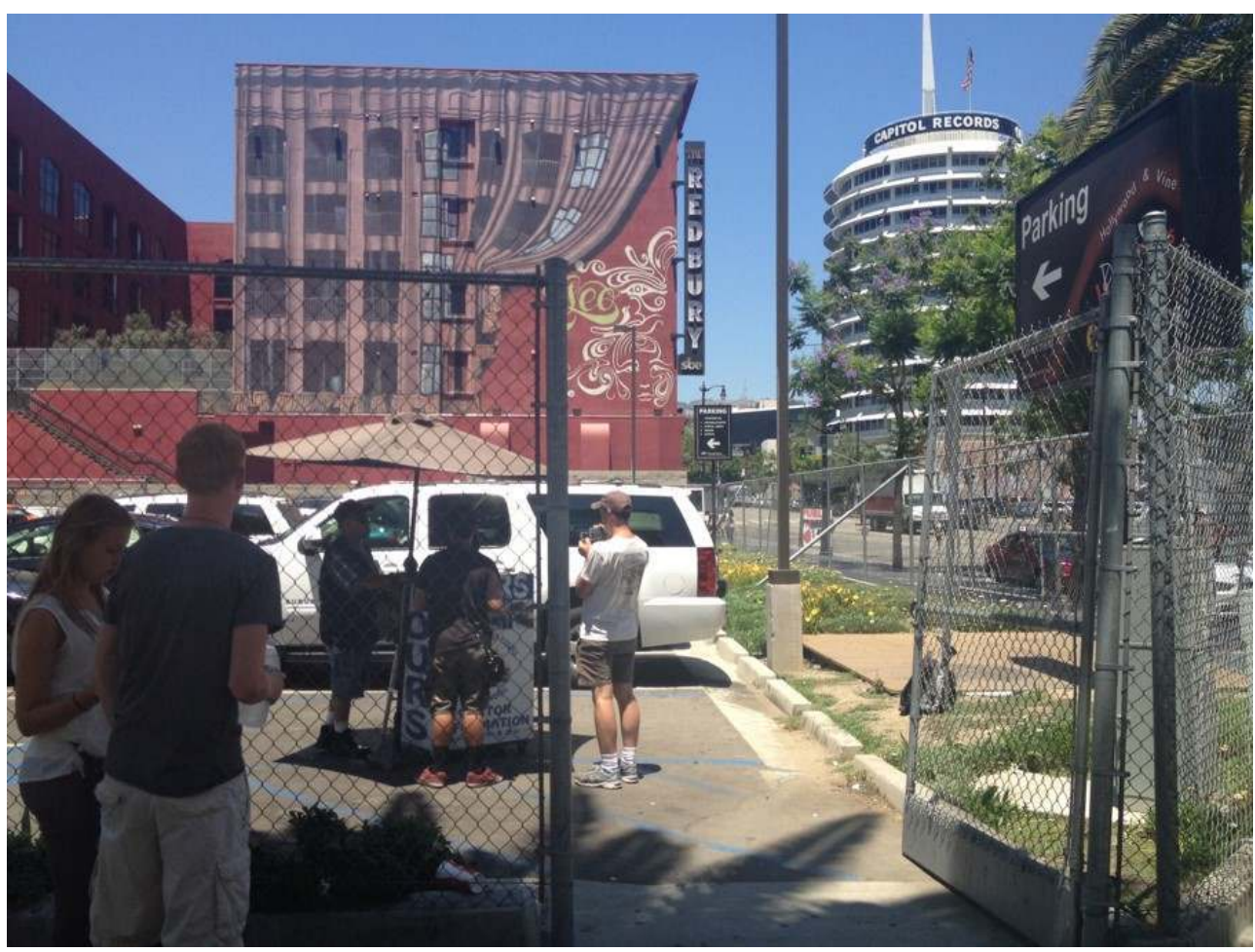

Crédit : auteur (juillet 2012, Hollywood Bd/Vine) 


\section{Controverses et délimitation de l'espace touristique}

7 Les services de bus touristiques ne sont ni nouveaux, ni récents. Catherine Cocks montre en effet comment cette technologie a été un outil permettant la découverte des métropoles américaines dès le début du $\mathrm{XX}^{\mathrm{e}}$ siècle :

like the railroads and hotels, the sight-seeing cars sought to provide an ideal social space removed from the urban masses (...) it was the modern, efficient way to get the job done right. (Cocks, 2001, p. 167)

Actuellement considéré comme un service populaire, utilisé par les "masses», ce dispositif était autrefois utilisé par les «élites» pour éviter toute promiscuité avec la foule. Si ce rôle de protection peut se comprendre dans le contexte des métropoles américaines de l'époque, c'est-à-dire celui d'un environnement hostile, peu « urbain » en quelque sorte toujours selon l'auteure (ibid.), qu'en est-il aujourd'hui?

9 Technologie spécifiquement touristique, destiné au sightseeing, à la découverte de l'espace urbain, le «bus touristique » est devenu un équipement indispensable, générique - dans la mesure où on le retrouve systématiquement - des métropoles: toute métropole touristique se doit d'avoir un service de bus touristiques. Il s'agit donc d'un indicateur non seulement de la touristicité d'un lieu, mais aussi du niveau de cette touristicité : il faut que l'espace urbain, le nombre "d'attractions touristiques" et le nombre de touristes soient suffisamment importants pour justifier la présence de ce service. S'interroger à ce sujet ne revient donc pas à restreindre l'argumentation à un cas spécifique (ici, celui de Los Angeles) mais à rendre possible une mise en perspective avec d'autres situations et s'interroger sur des logiques plus générales quant aux manières dont les individus habitent touristiquement les métropoles.

Pour autant, si le dispositif de bus touristiques est générique aux métropoles, on peut toutefois postuler que ce phénomène est particulièrement important à Los Angeles dans la mesure où deux types différents de services coexistent: des bus, qui proposent différents arrêts au sein de l'agglomération et correspondent au service que l'on retrouve classiquement dans les autres métropoles et des vans, dont le périmètre d'action se limite à Beverly Hills et à la découverte des villas des stars du cinéma. Cela participe d'une autre particularité qui complexifie la situation à Los Angeles : plus que l'ampleur considérable du phénomène (qui témoigne du succès de cette pratique) ${ }^{2}$, c'est sa manifestation ostensible sur le Walk of Fame de Hollywood Boulevard, que l'on observe rapidement en enquêtant sur place, qui interpelle tout à la fois les touristes (c'est un phénomène qu'ils ne peuvent pas ignorer, ce point sera abordé au cours de la réflexion) mais aussi le chercheur, obligeant ce dernier à le considérer pour en comprendre les ressorts et la manière dont il s'insère au sein des pratiques touristiques. Sur moins d'un kilomètre, ce n'est pas moins d'une vingtaine de compagnies, proposant aux touristes de faire une visite guidée, que l'on peut recenser sur cette partie du boulevard. Il n'y a que sur cette partie d'Hollywood Boulevard que le touriste est confronté à une telle situation. La concurrence est donc âpre entre les opérateurs, même si des collaborations existent à partir d'affinités individuelles : la « lutte des places » (Lussault, 2009) doit ici s'entendre au sens propre, avec des rixes entre membres de compagnies différentes (Martin, 2015). Pour autant, il existe une certaine stabilité et l'on retrouve chaque compagnie au même endroit d'un jour à l'autre, l'emplacement étant loué à un coût plus ou moins important (l'un des responsables m'a indiqué payer 3000 dollars par mois). En dépit de ce prix, il 
remarquait que ce placement était "pourri » (pour reprendre exactement son qualificatif) car se situant à l'extrémité est du Walk of Fame, au croisement avec Vine Street : la concentration des touristes y est beaucoup moins importante du fait du relatif éloignement par rapport aux principales «attractions " du lieu (situées à l'extrémité ouest), un grand nombre de touristes ne se promenant pas jusqu'à cette extrémité du boulevard.

Ces tensions sont le témoin de l'importance de cette activité et donc du caractère crucial de l'enjeu auquel elle répond. Les opérateurs sont confrontés à des réglementations par rapport à leur parcours et sont obligés de soumettre le tracé du circuit à certaines restrictions. C'est par exemple le cas à Beverly Hills, où s'effectue le transfert entre le " circuit jaune » et le « circuit rouge » : celui-ci se réalise dans une rue annexe, à l'arrièreplan des rues emblématiques de la municipalité. Malgré les réglementations, ces circuits provoquent un conflit d'usage de l'espace public : des associations de résidents de Beverly Hills et de Hollywood se plaignent du bruit occasionné par la fréquence du passage des bus touristiques. "On weekends, it's every 10 minutes", témoigne ainsi dans le Los Angeles Times le résident d'une rue régulièrement empruntée (Martin, 2011). Cette économie fait donc controverse, à différents aspects.

\section{Illustration 2. Circuit des bus touristiques de la compagnie Starline}

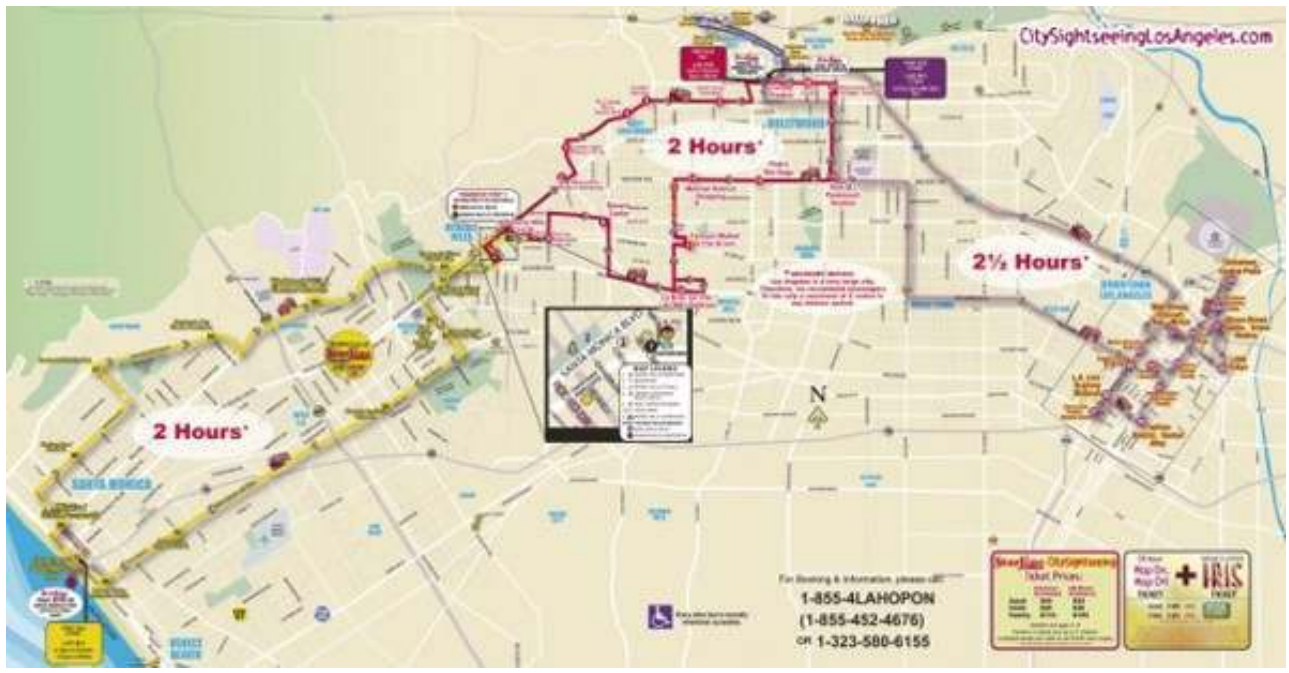

Source : www.starlinetours.com/ (reproduit avec autorisation)

Les bus touristiques ne font pas le «tour de la ville » mais seulement d'une (relativement) petite portion de l'aire urbaine - une trentaine de kilomètres - autour du Westside entre Downtown Los Angeles et Santa Monica (voir illustration 2). L'enjeu de délimitation de l'espace touristique se trouve ainsi également au cœur de ce système. Cette opération de délimitation nous renseigne sur les lieux que les promoteurs veulent désigner comme attraction, et de ce fait fournit une autre illustration de l'espace touristique de Los Angeles. Le choix d'une avenue au détriment d'une autre représente une sélection cruciale : en définissant un parcours préétabli à travers la ville, ces services indiquent d'une façon certaine aux touristes que « ce qui est intéressant à voir » est là et pas ailleurs. Les utilisateurs de ces services confient aux opérateurs le soin d'établir ce parcours et de les guider à travers les lieux les plus remarquables, sans qu'il s'agisse toutefois d'une confiance aveugle : un trop grand décalage entre le tour (visite) effectué et les attentes des utilisateurs nuirait au succès commercial de l'entreprise. D'ailleurs, le tracé ainsi défini 
corrobore l'ensemble des autres indicateurs permettant de cartographier l'espace touristique de Los Angeles et rend particulièrement visible le fait que ce dernier fonctionne de manière réticulaire, autour de nodalités (Soja, 2004) constituant un modèle polycentrique.

\section{Le bus touristique : une alternative à la voiture}

La première modalité de tour envisagée est celle réalisée par les bus touristiques, que l'on peut donc considérer comme le dispositif touristique classique des métropoles. Ils sont destinés à effectuer de longues distances à travers la partie de Los Angeles allant de Downtown L.A. à Santa Monica en passant par le Westside (voir illustration 2). Ils utilisent les grands axes de circulation et font de multiples arrêts, au niveau desquels les passagers peuvent monter/descendre. L'idée défendue ici est qu'ils servent non seulement à la découverte de la métropole "dans son ensemble ", mais aussi de moyen de transport à une manière (spécifiquement touristique) de gérer les distances. On retrouve très clairement cette idée de ne pas conduire dans la réaction sur Tripadvisor d'une jeune touriste anglaise ${ }^{3}$ :

We were thinking of not driving in L.A. to give ourselves a break - we're doing a lot of driving over 20 days and thought a bus tour might be nice way to relax for a couple of hours (hopefully in the sun on the top deck!)

Contrairement à la plupart des cas où l'utilisation de ce service est décidée sur le moment par les touristes, celle-ci est ici envisagée par la personne avant même le départ: cela tend à indiquer une préparation poussée du séjour en amont, avec un point de vue global sur le séjour et une prévision des conséquences inhérentes à ce genre de pratiques touristiques - cette capacité à se projeter découlant sans doute d'expériences passées. Cet extrait révèle le "versant stratégique » - pour reprendre l'expression de Michel de Certeau - des pratiques touristiques. On peut supposer qu'il en est de même pour Danny (australien, Perth, la trentaine), qui est déjà venu deux fois à Los Angeles. Même si l'agglomération n'est donc plus inconnue pour lui, sa pratique de la métropole étant informée par ses précédentes expériences, elle reste un lieu du hors-quotidien, imprégnée d'une forte altérité :

Yes, I don't have a car here, there is so much traffic, so it's probably easiest to walk. I take a bus, the "hop on/ hop off » tour. It was ok, because we stopped in many places, it was informative because they explained a lot of things, I also wanted to get to the beach so it was ok. I take the bus because it takes the main routes I wanted to go to. I wanted to go to Santa Monica, in Downtown... so I just paid for one pass. I didn't go by myself and get lost... but it's stopping and starting all the times. If it was a direct route it would be easy. It's not so far, it's just because of the traffic.

La prédominance de la métrique automobile, et surtout la circulation très importante sont des éléments handicapants du point de vue de cet individu pour habiter touristiquement la métapole. Malgré sa connaissance du lieu, la configuration spatiale de Los Angeles représente toujours une épreuve, notamment par rapport à la question de la gestion des distances entre les lieux. Il estime ne pas avoir la maîtrise technique suffisante pour conduire à Los Angeles (c'est une conduite inversée par rapport à l'Australie) et pour se repérer. Le fait de ne pas se sentir capable de conduire l'incite à choisir le bus touristique comme moyen de déplacement, plutôt que de louer une voiture ; et s'il n'opte pas pour les transports en commun - il indique ne pas utiliser le métro parce qu'il préfère « to stay out on the street »-, dont le pass journalier est de seulement quelques 
dollars (contre une quarantaine de dollars par personne pour le ticket d'un bus touristique) mais objectivement plus lents du fait du nombre d'arrêts plus important, et moins adéquats pour le sightseeing, on peut faire l'hypothèse que c'est parce qu'il n'a pas l'habitude de les utiliser au quotidien, qu'il n'en a pas la maîtrise. Cet exemple illustre le fait que certains touristes peuvent encore faire appel à ce genre de services même en connaissant déjà la métropole.

Ce n'est pas du tout le cas de Paul-Henri, qui fait l'expérience de Los Angeles pour la première fois dans une situation particulièrement atypique : âgé de 21 ans, né à Paris, il est à Los Angeles pour seulement 10 heures, dans le cadre d'une correspondance entre son vol arrivant de Montréal - où il était en vacances - et celui vers la Nouvelle-Zélande, où il doit retrouver son amie (avant de retourner à Sydney, où il étudie) :

J'ai 11 heures d'attente à l'aéroport, donc là j'en profite... J'ai pris un bus... Un bus qui fait le tour, Hollywood, Venice Beach et tout... Pour l'instant, c'est que le début, mais vu que ça fait 100 kilomètres de long cette ville, j’ai préféré prendre un bus qui fait tout direct plutôt que de me balader tout seul en taxi quoi ! (...) Je suis arrivé à l'aéroport. Je suis allé à un point information. Ils m'ont dit d'aller au Hilton du LAX (...) Après... ça à l'air vachement dur de visiter Los Angeles par soi-même ; ça a l'air tellement immense... En avion, quand on arrive à l'aéroport, on met quinze minutes pour voir la ville en entier (...). Moi je trouve que c'est pas mal, pour les gens dans mon cas en tout cas. Pour quelqu'un qui reste deux semaines, je trouverai ça un peu stupide. Parce que c'est vraiment... c'est vraiment le piège à touristes quoi. Enfin, moi je ne suis pas trop pour les trucs touristiques à la base, donc c'est une première fois le bus! Mais pour quelqu'un qui a deux jours, ou même comme moi un aprèsmidi, c'est super et c'est pas très cher en plus (...). Mais sinon, j'aime pas passer pour un touriste! J'aime pas avoir un gros sac à dos, des bottes de randonnée et tout le bordel... J'aime bien arriver mine de rien quoi, et puis... je suis pas forcément dans l'ambiance où j'ai envie d'être avec plein de mecs qui sont là en vacances en même temps, tu vois... Tu fais un tour de bus à Los Angeles, tu fais un tour de bus à Montréal ou à Rio, ben t'es toujours avec les mêmes gars... des Européens, des Japonais... et c'est juste le décor qui change, tu vois? Alors que si je peux parler avec un mec d'ici, ça change complètement...

Quand il atterrit à Los Angeles, ce touriste n'a ainsi encore rien prévu, ne possède ni guide ni carte et n'a pas effectué de recherche sur Internet. Il n'a donc aucun renseignement sur ce qu'il y a à faire ou à voir, mis à part les représentations qu'il a probablement de Los Angeles à travers les médias. Sur cette base, une évaluation rapide de la taille de l'agglomération lui permet d'avancer que celle-ci constitue une difficulté importante pour visiter cette métropole par lui-même. Aussi, pour effectuer malgré tout une visite express, le bus touristique lui apparaît comme la métrique la plus adéquate par rapport à sa situation et à cette configuration spatiale : elle a l'avantage d'être plus développée et efficace que les transports en commun réguliers, moins coûteuse que le taxi et moins difficile à maîtriser que l'automobile. On saisit bien ici comment le bus touristique est une technologie spatiale qui permet de compenser la faiblesse des connaissances sur le lieu: en utilisant cet outil, Paul-Henri n'a pas besoin de chercher les lieux les plus emblématiques de la métropole, de trouver par quel moyen de transport il peut s'y rendre, ni comment organiser son parcours entre ces différents lieux; ces trois aspects sont intégrés dans la prestation. Il effectue un arbitrage in situ (par rapport à la configuration spatiale, au temps disponible, au coût financier) qui dénote une certaine capacité d'adaptation. Car même s'il estime que ce n'est pas cher, on comprend malgré tout que " ça lui en coûte " quand même d'utiliser ce service : cette manière de faire va à l'encontre de ses convictions, de la vision caricaturale qu'il peut avoir du touriste et de ce 
qu'il ferait dans d'autres circonstances. C'est uniquement le caractère urgent de son séjour qui lui permet de justifier et trouver une certaine rationalité à sa pratique, de «sauver la face ».

Illustration 3. Bus touristique sur le Walk of Fame

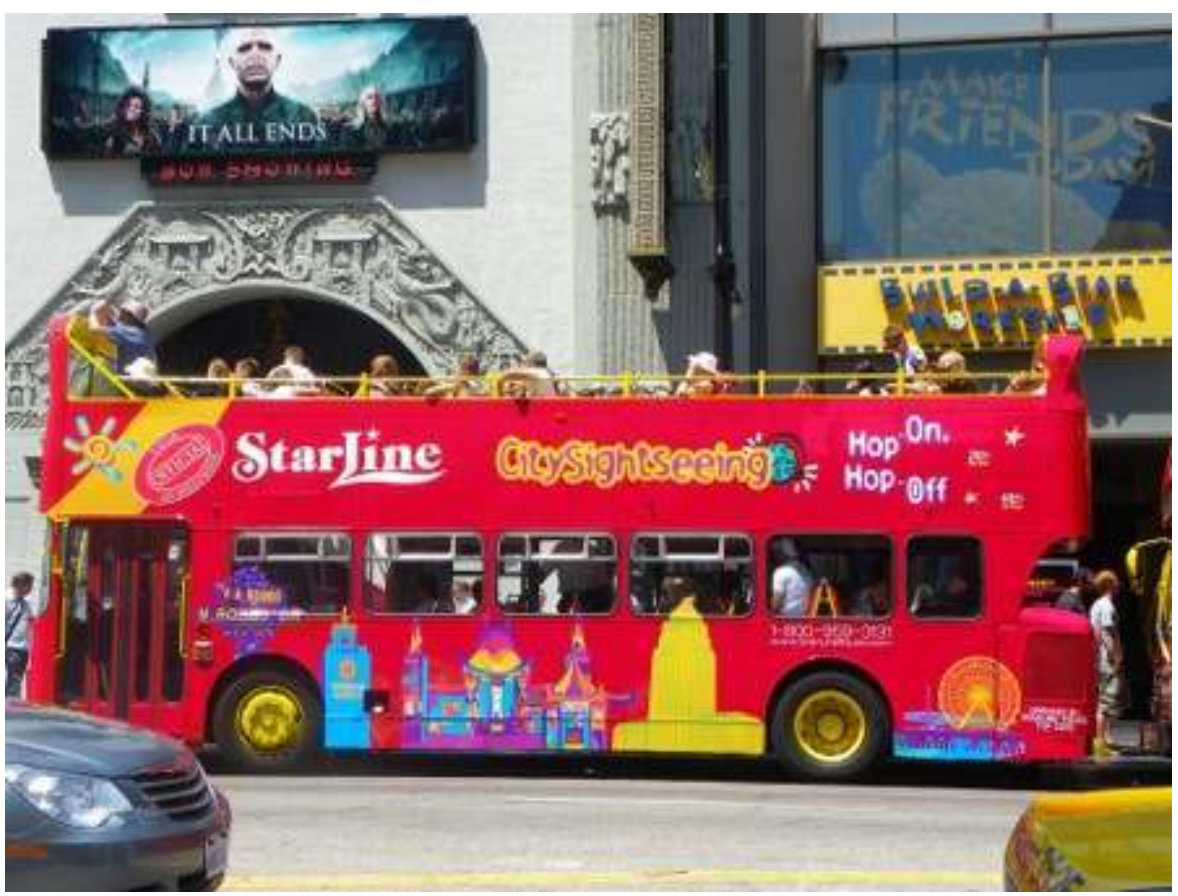

Crédit : auteur (juillet 2011)

18 Il apparaît explicitement que les bus touristiques servent à se déplacer à travers la métropole, comme une façon de gérer les distances entre les différents lieux touristiques de Los Angeles. Ces trois touristes n'ont, pour des raisons différentes, pas de voiture : le bus remplace alors cette dernière. Par rapport aux autres modes de transport possibles, il a un double avantage : d'une part, il fournit une sélection des endroits considérés comme dignes d'intérêt pour les touristes; d'autre part, c'est un véhicule spécialement adapté pour le sightseeing car, disposant d'un deuxième étage avec un toit ouvert, il permet d'avoir une vue sur la ville sensiblement différente de celle que l'on peut avoir avec un bus régulier par exemple. Ces exemples ne sont pas des exceptions, au contraire. Comme il a été possible de l'observer à l'occasion des différents tours effectués dans ces bus, la plupart des touristes descendent aux différents arrêts proposés : il s'agit bien d'un moyen de déplacement entre les différents lieux, d'une métrique, d'une façon de gérer les distances entre différents lieux, qui remplace l'utilisation des transports publics ou de la voiture, et non juste un moyen de voir la ville. C'est une véritable alternative à la voiture pour ceux qui ne peuvent pas ou qui estiment ne pas avoir les compétences ou les connaissances suffisantes pour visiter par eux-mêmes.

19 Constatons enfin que parmi les options envisagées par Paul-Henri, la possibilité de louer une voiture pour quelques heures n'est pas évoquée, comme hors de propos. Cela ne va pas de soi pourtant. Pour mettre en perspective ces discours et cette manière de faire avec l'espace, il n'est pas inutile d'envisager le cas de « Swdke » ${ }^{4}$ qui ne passe lui aussi que quelques heures à Los Angeles : 
I have an afternoon flight out of LAX, but wanted to do some tourist stuff. Taking my Mom on a holiday, said she wanted to take a tour of Hollywood. I asked specifically what she wanted to see, and all she can think of is movie star homes. But, wants to see other stuff too. She's been to California and L.A. a good number of times, so I am not sure why she can't figure out what else she wants to see. Anyhow, I figured maybe take the Starline 2 hours movie star home. After the tour, have a quick lunch at Pinks. Then, head to Paramount Studios for a studios tour at 1 p.m. After the tour, then head back to airport. I'll have a rental car which I need to return and take the rental shuttle to the airport. If I depart Paramount studios around 3:15 p.m., and need make my way to LAX, drop off the car, do you think I should be okay to be in the terminal by 5 p.m.?

Cet extrait permet d'illustrer l'importance de la maîtrise de techniques dans les manières de faire avec l'espace des individus : il est tout à fait possible d'habiter touristiquement Los Angeles pour quelques heures avec une voiture si l'on a (ou estime avoir) les compétences suffisantes pour le faire. Ce touriste indique pourtant qu'il souhaite également effectuer un tour: il s'agit ici de la seconde modalité présente à Los Angeles, qui peut correspondre à une pratique touristique au sens strict mais aussi à un outil pour compenser la faiblesse de compétences ou de connaissances.

\section{Le van touristique : se faire balader}

Parallèlement à ce système conventionnel, classique pour les métropoles touristiques, la seconde modalité de circuit est spécifique à Los Angeles. Il s'agit d'un circuit thématique correspondant au «star homes tour », dont l'objectif principal n'est plus de se déplacer à travers la métapole, mais d'observer les villas des célébrités à Beverly Hills. Par rapport aux bus touristiques abordés précédemment, l'ampleur de ce circuit, d'une durée de deux heures, est géographiquement plus restreinte, se limitant à Hollywood et Beverly Hills. Par ailleurs, il n'y a pas d'arrêts prévus : les touristes ne peuvent pas descendre pendant le parcours. Ce service n'est donc pas utilisé pour aller d'un point à un autre. Afin d'accéder aux luxueuses villas et de faciliter la circulation dans les petites rues sinueuses de ces quartiers huppés, les véhicules utilisés sont également de plus petite taille : il s'agit plutôt de vans que de bus. Les circuits des vans touristiques reposent sur un fonctionnement plus flexible: les axes de circulation empruntés par le tour ne sont pas aussi précisément définis que pour les bus, le conducteur qui en a la responsabilité pouvant faire varier le parcours en fonction des conditions de circulation, même si l'on peut supposer une certaine régularité afin de ne pas devoir changer les commentaires prévus. Un autre élément important est à prendre en compte : contrairement à ceux qui empruntent les bus touristiques, une très grande majorité des utilisateurs des vans ont également loué une voiture. Pourquoi alors dépenser à nouveau de l'argent pour utiliser ce service? 
Illustration 4. «A drive-in tourist gaze »

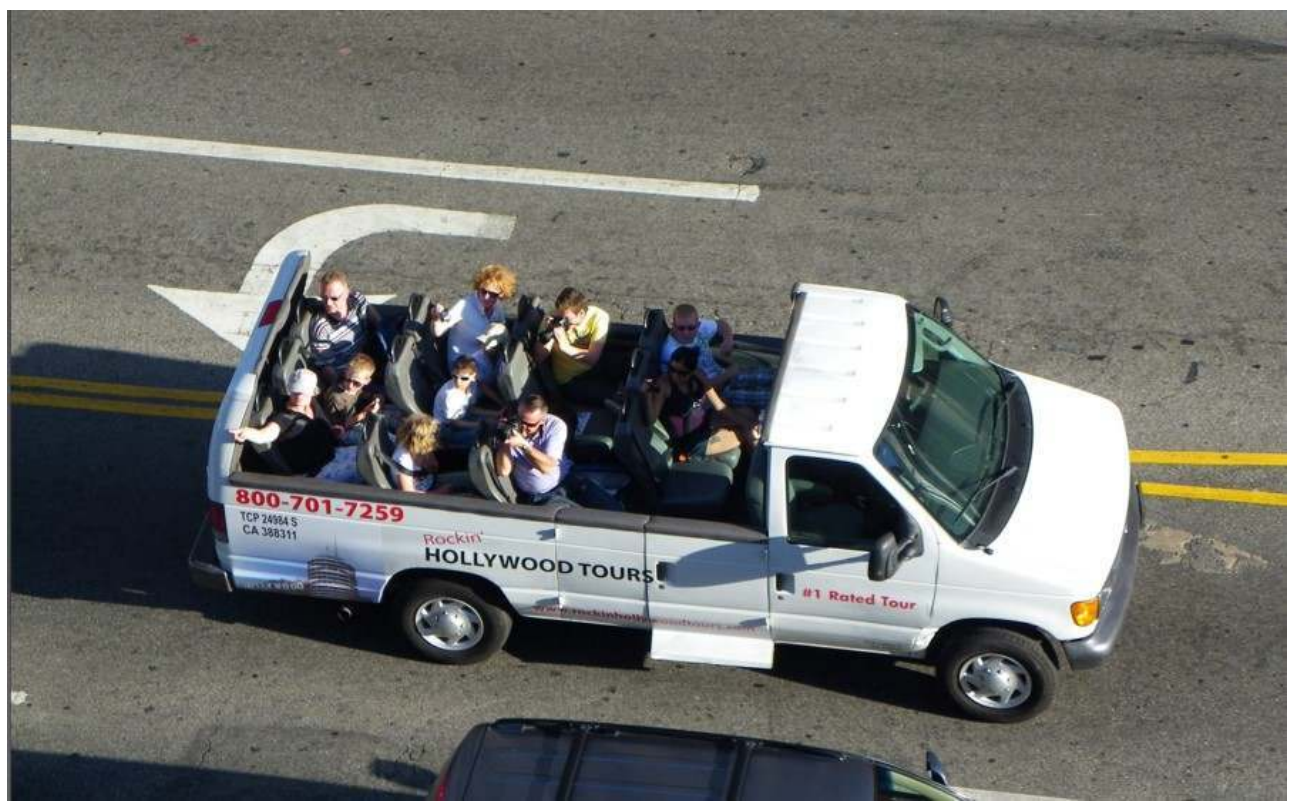

Crédit : auteur (juillet 2011, Hollywood Bd)

Pour aborder cette question, nous décrirons dans un premier temps les logiques de ce que l'on peut considérer comme une pratique touristique à part entière, qui constitue une expérience touristique adossée à la dimension cinématographique. Dans un deuxième temps, l'hypothèse développée est que l'utilisation de ce service par les touristes va audelà de la simple pratique, mais correspond aussi à une manière de gérer les distances et de compenser la faiblesse des maîtrises techniques et/ou des connaissances. Laissons tout d'abord à une touriste ${ }^{5}$ le soin de décrire cette pratique :

I was in L.A. last week and did the Stars Homes tour with Starline. They seem to be the most popular (though there are certainly others nearby). There are plenty of small booths around the Hollywood and Highland Center where you can book. I stopped at a booth at 10 a.m., and was on the tour at 10:15 a.m. It was a 2 hours tour; not only did we see the homes, the guide also provided narration as we left Hollywood and traveled along Mulholland Drive and the Sunset Strip area. Yes, it's a touristy thing to do, but I found it fun! I can see why some people might make that comment about the gates, as you do see some gates and shrubbery. I would say it was about 50/50 (gates vs. actual houses). However, the guide I had was good about driving around so that we could see as much of the house as possible, even if it was from a distance. I thought it was worthwhile, as the drive through some of the neighborhoods of Beverly Hills was enjoyable in and of itself. Being my first visit to the area, I thought the rest of the commentary about the neighborhoods and Hollywood added a lot to my visit and also gave a good overview of the area.

S'il s'agit là d'un commentaire positif, les avis sont partagés, avec des appréciations plus négatives comme j'ai pu le constater dans certains de mes entretiens : « it's not very good, you don't see anything» constate Almorena (espagnole, 24 ans, voyage avec son compagnon), pour n'en citer qu'un exemple. Cette pratique touristique correspond à la mise en scène d'un quartier huppé strictement résidentiel : même si les villas - plus exactement leurs portes d'entrée et leurs clôtures (voir illustration 4) - que l'on voit durant le tour sont d'un haut standing, il s'agit d'un espace urbain d'une monofonctionnalité totale, où il ne se passe pas grand-chose et où l'on ne croise pas grand 
monde, si ce n'est les vans des autres compagnies. Il est donc possible d'envisager, premièrement, cette modalité de circuit comme une pratique touristique en soi et spécifique à Los Angeles, comme on le constate à travers l'affirmation de Cathy (australienne, Brisbane, 27 ans, voyage avec deux amies) : « it's just something you have to do when you come to Hollywood». De son point de vue, il s'agit d'une pratique incontournable : le tour des maisons des célébrités fait pleinement partie de l'expérience de Los Angeles. C'est d'ailleurs par cet aspect (au même titre que la visite des studios et le Hollywood Sign) que l'on mesure combien la dimension touristique de Los Angeles s'appuie et est indissociablement liée à la dimension cinématographique qui fait la réputation de cette métropole à l'échelle mondiale (Monnet,1999).

L'autre principale caractéristique qu'il convient de retenir par rapport à ce système est donc qu'une très grande majorité des touristes effectuant ce type de tours dispose également d'une voiture : le fait de payer ( 20 dollars, plus 10 dollars de pourboire pour le conducteur) pour un circuit dans un van touristique ne se substitue pas à l'usage d'une automobile mais vient s'y ajouter de façon complémentaire. C'est en cela que l'on peut considérer, dans un premier temps, l'utilisation de ce service comme une pratique «en tant que telle » et non comme un mode de déplacement. Mais cet intérêt à découvrir les maisons des célébrités est-elle la seule raison qui pousse les touristes à faire ce tour ? Il est possible de le faire par soi-même, des cartes, supposées renseigner la localisation des propriétés des célébrités, étant par ailleurs en vente en libre-service. Dès lors, pourquoi les touristes utilisent-ils ce service ? L'hypothèse défendue ici est qu'il s'agit d'un moyen pour les utilisateurs de compenser une faiblesse en termes de maitrise de techniques (difficulté à conduire, à se déplacer par soi-même à Los Angeles) et de connaissances (ne pas savoir où aller ni quoi faire).

\section{Pallier un manque de compétences/ de connaissances}

Une mise en récit de l'espace urbain. L'un des éléments de justification régulièrement revenu lors des entretiens quant à l'utilisation du van touristique a été celui de disposer d'une mise en récit de l'espace urbain : un certain nombre de touristes semble désirer avoir une expérience de la ville mise en récit. D'une certaine façon, certains touristes souhaitent en savoir davantage sur la dimension historique. C'est ce que l'on constate dans la réponse apportée par François : «[...] parce qu'il faudrait connaître les endroits précis à visiter. Là, on va voir les choses les plus importantes et ça nous évite de conduire. Ce sera expliqué aussi avec l'histoire des stars. » Il semble ainsi possible de renverser le discours de dénigrement classique sur les bus touristiques, comme l'archétype d'un tourisme de masse, «idiot ", pour au contraire l'interpréter comme une volonté de ne pas traverser passivement le paysage urbain mais d'en avoir des éléments de compréhension, des commentaires qui permettent de donner une épaisseur supplémentaire à leur visite même si les propos tiennent souvent plus du registre du mythe que du fait historique - en faisant un lien entre des monuments et un récit. 


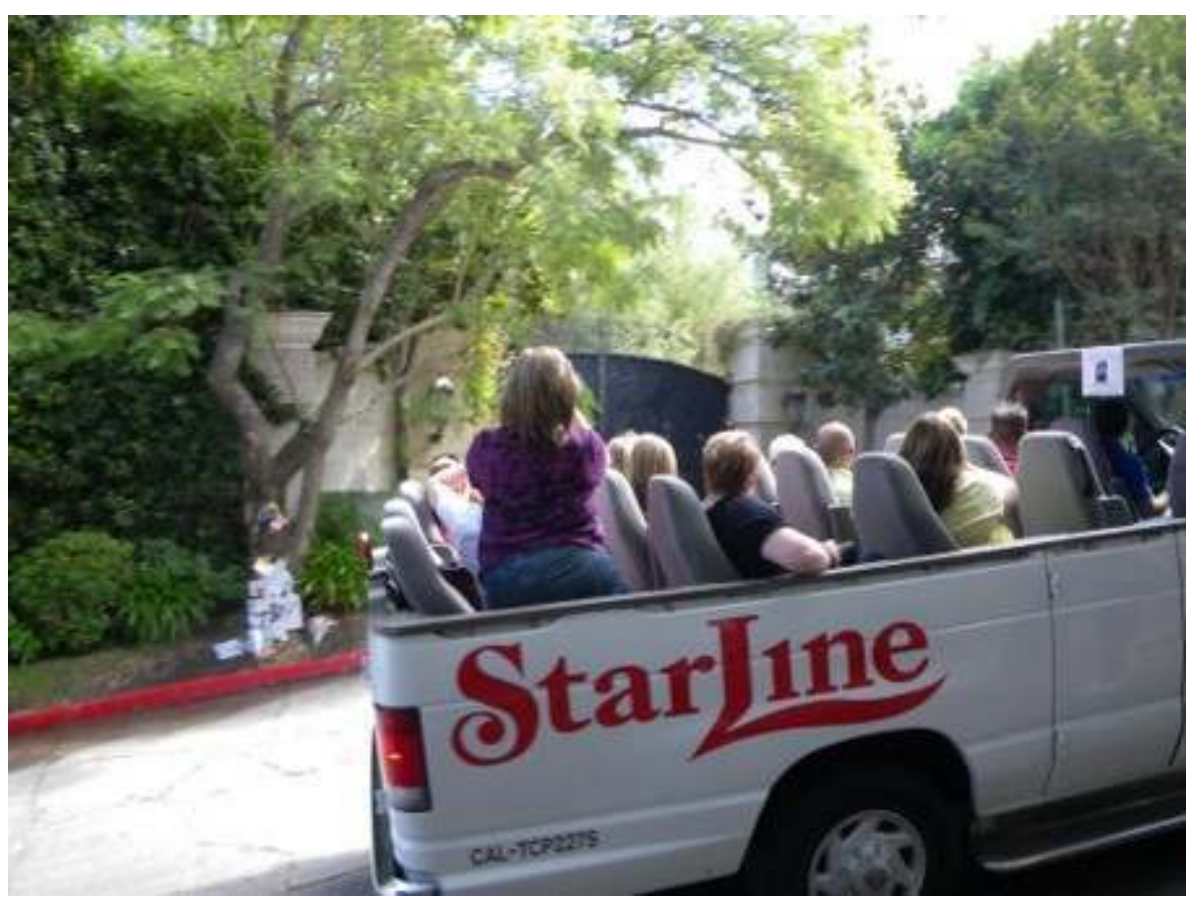

Crédit : Kevin Boyd (reproduit avec autorisation), www.flickr.com/photos/beryllium/3989589892/

Il faut également prendre en compte le fait que certains touristes font ce tour sans particulièrement avoir envie de voir les «maisons des stars» mais plus largement pour découvrir l'environnement urbain de cette partie de la ville. Les propos de Jérémy (français, la trentaine) confirment et complètent ces éléments :

On fait ce tour parce que c'est plus pratique qu'en voiture. On ne reste qu'une journée ici et on ne connaît pas trop le quartier, ce qu'il y a à voir... On a été abordé sur le Boulevard et on a été convaincu. C'est pour voir le plus possible, on ne peut pas perdre trop de temps. On veut voir Hollywood, Beverly Hills, quelques maisons de stars, mais sans plus. Au début, on avait peur que ce soit une arnaque, mais au final on le fait quand même. Ici, on nous accoste tous les vingt mètres pour nous vendre des trucs...

They told us in the street. Contrairement à ce que pouvait laisser entendre le point de vue de Cathy, qui faisait de ce type de tours un « incontournable », il ressort de l'enquête que le recours à des tours guidés n'est pas planifié par une grande majorité des touristes. Les différents extraits nous informent tous en effet du caractère spontané, imprévu de cette pratique : les touristes décident de le faire dans la rue. L'entretien avec Jérémy révèle même la réticence initiale, partagée elle aussi par la plupart des utilisateurs, par rapport à la manière d'être abordés. Comment dès lors comprendre cette décision, subite et initialement non désirée ? Les touristes se sont-ils « laissés avoir » par les sollicitations constantes des vendeurs, par "l'enchantement» du lieu pour faire référence à l'expérience de Yves Winkin (1997) ? C'est ce que tend à indiquer ce passage d'entretien réalisé avec Linda (journaliste française, la trentaine, sans voiture) qui participe à un tour en van « malgré elle » en quelque sorte :

On s'était organisé une journée pour aller sur les grands boulevards, faire Sunset Boulevard... et en fait on s'est rendu compte qu'en partant à 14 heures de Santa Monica on est arrivé à 17 heures. C'est hallucinant. On a mis trois heures. On a pris le bus avec une correspondance à Fairfax. J'étais estomaquée du temps qu'on mettait à traverser la ville. En plus, il y en a plein qui t'alpaguent en te proposant 
un circuit de deux heures en passant par Rodeo Drive etc. Aller voir les murs des stars, ça ne m'intéresse pas. Passer devant chez eux et voir leur maison, non merci. Arrivés sur Hollywood Boulevard, tout de suite ils te proposent tous des tours... En même temps, on réfléchissait parce qu'on piétinait et finalement on a décidé de le faire. On a fait un tour de deux heures sur Rodeo Drive, Beverly Hills... Tu te dis que c'est un gain de temps précieux mais ce n'est pas forcément le cas. Sur le prospectus, ils t'endorment un peu: ils montrent le signe d'Hollywood en grand alors tu penses que tu vas aller le voir de tout près, mais en réalité ils t'emmènent sur une colline à quelques minutes d'Hollywood. Ensuite, j'ai demandé quand est-ce qu'on allait aller sur la montagne, mais il m'a dit qu'on n'y allait pas. On nous a récupérés dans le bus à Hollywood Boulevard et du coup ils considéraient qu'on n'avait pas à faire le boulevard. On s'est fait balader pendant une heure et demie. On a fait des petites rues, ça c'était assez sympa. Mais le côté paillettes etc., ça ne me plait pas... On ne voulait pas prendre un car de touristes, forcément. J'ai vu que Starline avait des grands bus à deux étages mais aussi des pickups avec une dizaine de personnes à bord, on trouvait ça un peu plus intimiste. Les autres bus ça fait vraiment industrie à la chaîne. (...) Je n'aime pas le tourisme de masse comme ça, ça fait un peu zoo, ça dénature beaucoup la visite. C'est du paraître, il n'y a rien d'authentique là-dedans. Mais c'est à l'image de ce qu'est le lieu. J'ai été déçue par le tour mais il correspond assez bien à l'image qu'on peut se faire d'Hollywood.

On constate explicitement à travers ce large extrait combien la configuration spatiale - la taille et les distances de l'espace urbain - est handicapante pour cette touriste : c'est la difficulté à maitriser les métriques qui l'oblige à recourir à un service qu'elle n'apprécie pas (à l'instar de Paul-Henri), comme on peut le constater à travers le discours péjoratif qu'elle utilise pour le décrire. Il s'agit d'une pratique imprévue, indésirée presque, mais qui s'impose au groupe par l'insistance des vendeurs : à peine arrivées à Hollywood, après trois heures de trajet, et sans réellement marcher sur le Walk of Fame, ces touristes embarquent pour un circuit de deux heures et passent donc une grande partie de leur journée dans les transports (si l'on tient compte du trajet retour). La stratégie de vente des rabatteurs est d'ailleurs explicitement focalisée sur cette question des déplacements, en insistant dans leurs discours sur les très longues distances à parcourir (j'ai souvent entendu le mot « huge » dans leurs propos pour tenter de convaincre les touristes sur le caractère irréalisable d'une visite par eux-mêmes). Il faut à ce titre remarquer que Linda, et on peut supposer qu'elle n'est pas la seule, semble confondre les deux systèmes de tours , croyant que le service proposé par les vans est identique à celui des bus, avec comme seule différence la taille du véhicule. 


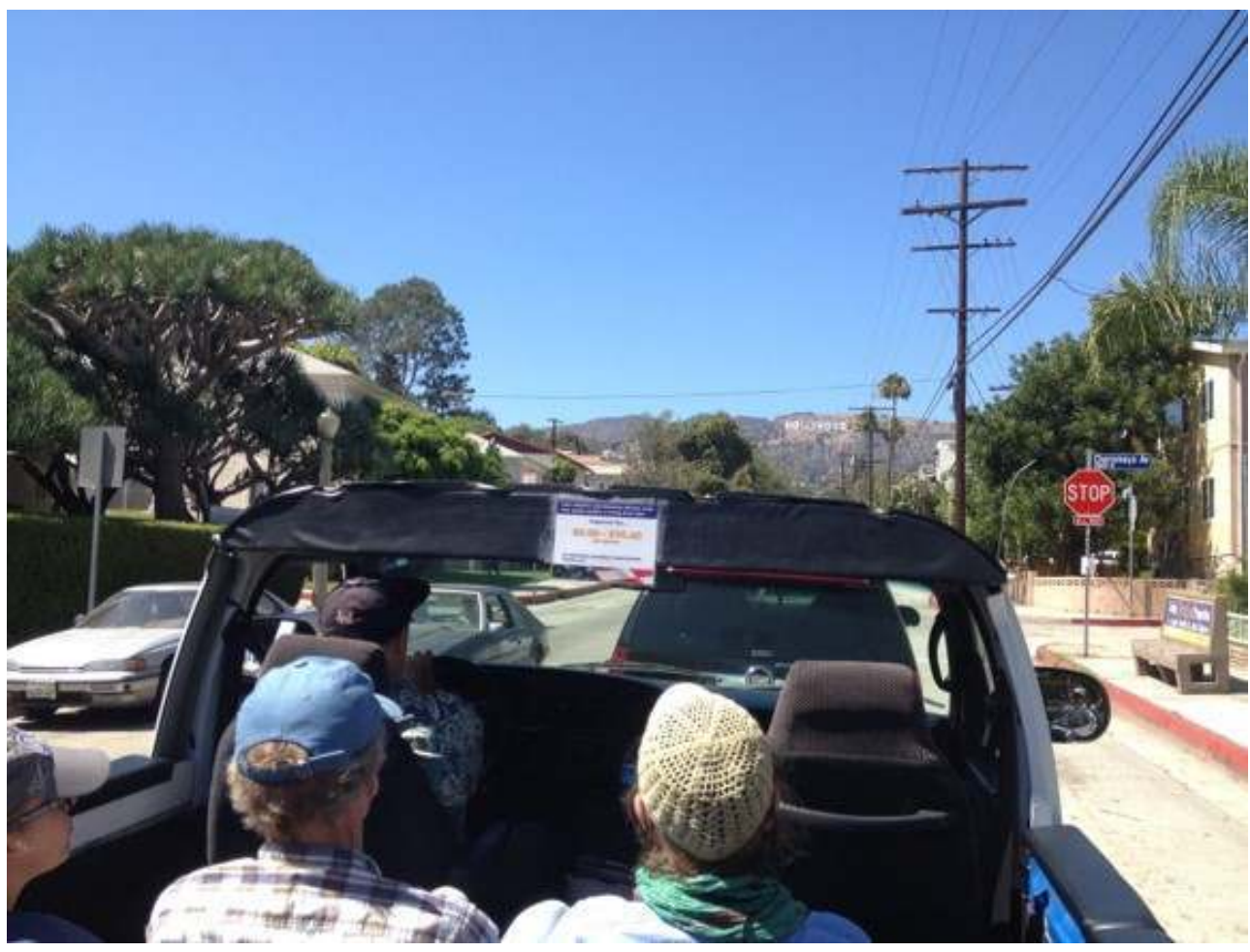

Crédit : auteur (juillet 2012)

29 On peut, plus largement, considérer l'utilisation du van touristique comme résultant d'un arbitrage effectué in situ par les individus : il s'agirait de la manifestation de l'évaluation des distances et de la complexité de la configuration urbaine mesurée par ces derniers. Ce serait alors l'une des expressions du versant tactique des manières touristiques de faire avec de l'espace, qui relèvent alors pleinement du domaine de l'improvisation et de l'adaptation à une situation singulière, imprégnée par l'automobile.

Because we don't want to drive a lot est en effet une réponse qui revient fréquemment pour expliquer cette pratique. Une part très importante des touristes internationaux (tout du moins européens) séjournant à Los Angeles font un tour de la Californie de deux ou trois semaines, avec de longs trajets entre les différentes étapes du parcours. L'utilisation d'un van peut alors se concevoir comme une tactique choisie par les touristes pour se reposer. Il faut pleinement intégrer le côté recréatif de la pratique touristique d'une métropole pour apprécier l'ensemble des dimensions intervenant dans le choix d'utiliser un tour en bus : se «faire balader » autorise un relâchement que ne permet pas la conduite. Qui plus est, les bus et vans sont des véhicules spécialement adaptés au sightseeing, avec leur toit ouvert, ce qui est rarement le cas des voitures de location. Cet aspect ressort clairement des propos de Gilles et Françoise (canadiens, la quarantaine, voyagent avec deux enfants) : «On fait un tour en bus touristique pour éviter de conduire. On a loué une voiture à San Francisco, on fait un voyage en Californie, on finit à L.A. On espère qu'ils vont nous expliquer les choses et donner des informations». Si cette famille veut éviter de conduire c'est sans doute moins parce qu'il est difficile pour eux de circuler à Los Angeles mais c'est surtout pour « lever le pied ». Il faut véritablement penser la pratique touristique à Los Angeles comme une courte étape d'un circuit touristique beaucoup plus vaste, qui peut entraîner une lassitude vis-à-vis de la conduite. La prise en compte du 
temps à disposition est d'ailleurs un paramètre qui intervient souvent dans la décision des touristes, comme le souligne ces différents petits extraits d'entretiens :

We decided to do this on the street. They said we were going to see a lot in a few time. And it's not expensive. (Jim)

Because they tell story about the places, and we don't have to drive and find the directions. We don't have a lot of time to visit. (Marine)

C'est pour ne pas perdre de temps, aller à l'essentiel. On ne reste que deux jours ici. (Marc et Carine)

To see a lot in a few time. La volonté de ne pas perdre de temps est en effet un autre argument régulièrement avancé par les utilisateurs pour justifier cette pratique. Ils doivent faire face à une situation paradoxale : ils restent peu de temps dans une métapole où le moindre déplacement en demande beaucoup, l'étalement de l'espace touristique impliquant des temps de parcours longs, qui plus est lorsqu'on ne connaît pas l'agglomération. L'usage d'un tel service répond alors à un souci d'efficacité. Cette pratique du tour en van s'insère dans une évaluation de la meilleure façon de faire avec cet espace de la part des touristes, par rapport à leurs compétences pour se déplacer et leurs faibles connaissances du lieu. On peut faire un lien entre cette recherche d'efficacité et la faiblesse des compétences (la maîtrise de techniques) et/ou de connaissances d'un certain nombre de touristes utilisant les vans touristiques. Les enquêtes ont en effet permis de constater qu'une grande partie d'entre eux indiquaient ne pas savoir où aller, ne pas savoir ce qu'il y a "d'intéressant » à voir ou à faire à Los Angeles :

Because we want to see L.A., and we didn't know where to go. (Aaron)

It's easier for us. It takes us to see the places. We didn't organize at home. And we've never been on a tour on a bus. (Gil et Nathia)

Because it's easier, it takes us directly. It's not easy to move around in L.A., we don't really know where we have to go. (Chris)

Because we don't know where the places to see are. It's easier. (Samantha)

Because we don't know around. We have a guidebook, but it's not easy to follow it. (Luis)

We don't know where we have to go. It's easier. Les touristes expriment explicitement choisir une option qui leur permet de faire avec l'espace plus facilement, d'atténuer la complexité des épreuves spatiales : ils acceptent de payer pour un circuit en van parce que c'est le moyen le plus facile pour habiter touristiquement Los Angeles. Il s'agit d'un indice dénotant la difficulté que peuvent avoir certains touristes à se déplacer et se repérer dans l'espace urbain, et donc à réaliser leurs pratiques touristiques. L'utilisation de ce mot, easier, loin d'être anecdotique, signifie donc qu'une grande partie des touristes utilisant ce service considèrent que c'est compliqué de découvrir Los Angeles par soimême, même lorsqu'on possède un guide touristique ${ }^{7}$. Mais quelles sont les raisons de cette difficulté ? Le principal motif qui apparaît dans les entretiens est que les touristes ne savent pas " où il faut aller » et "ce qu'il y a à voir ": il s'agit explicitement d'une faiblesse en termes de connaissances, compensée par l'utilisation d'une technologie spatiale au moyen de la mobilisation du capital économique. Les touristes s'en remettent explicitement à ces opérateurs, à ces intermédiaires pour leur dire et leur montrer les lieux remarquables d'une partie de la métropole : c'est ce que traduit la présence dans leurs discours des expressions "aller à l'essentiel ", "les choses importantes». Cela indique implicitement que ce n'est pas « Los Angeles » qui est à découvrir, à visiter, dont il faut faire l'expérience, mais quelques lieux précis, certains monuments - les marqueurs pour reprendre la terminologie de MacCannell (1976). Il faut "voir ce qui est à voir ", c'est-à-dire les lieux reconnus socialement, par les autres touristes, comme étant dignes 
d'intérêt. Certains touristes ne prennent ainsi pas seulement un van pour voir les maisons des célébrités, mais plus largement pour découvrir Los Angeles.

Le circuit proposé par les vans touristiques répond à une attente largement partagée par les touristes de voir les résidences des célébrités. S'il est possible de faire par soi-même un tour de Beverly Hills pour voir les villas, on peut avancer l'idée que c'est moins évident, moins « riche» et moins confortable qu'en se "faisant balader » à travers ce quartier de la métapole. Utiliser un van touristique semble être le moyen le plus adéquat et efficace pour réaliser cette pratique. On a toutefois pu constater que l'utilisation des vans touristiques est un indicateur de la difficulté d'une certaine partie des touristes à habiter Los Angeles, à faire touristiquement avec cet espace : ces utilisateurs indiquent souvent que la configuration urbaine ne se prête pas à un investissement facile. Pour un certain nombre de touristes, la métrique automobile est handicapante pour habiter touristiquement. Cela correspond donc plus à une réponse à un besoin qu'à un simple élément de confort. Parallèlement, on peut relever que cette difficulté s'accompagne de (est due aux?) faibles connaissances dont dispose ces individus sur Los Angeles: nombreux sont ceux qui indiquent ne pas savoir quoi voir, quoi faire. De façon synthétique, on peut ainsi avancer que parmi l'ensemble des touristes abordés par cette enquête, la plupart de ceux effectuant un circuit expriment ne pas disposer d'une maitrise de techniques et des connaissances suffisantes, alors qu'inversement, ceux disposant des compétences et des connaissances suffisantes n'ont pas effectué de circuit ${ }^{8}$.

\section{Conclusion}

Reformulons l'argument central de cet article d'une autre manière : parmi l'ensemble des touristes envisagés dans le cadre de l'enquête, ceux pour qui ce n'était pas compliqué de se déplacer à Los Angeles (en voitures ou en transports en commun) n'ont pas pris de bus ou de vans touristiques. Les deux principales modalités de tours évoquées, que l'on peut croire au premier abord i) radicalement différentes dans leurs objectifs, tout en constituant chacune ii) une pratique touristique en tant que telle, sont en réalité d'une utilité de la toute première importance, répondant à une même demande de la part des touristes qui les utilisent : faire plus facilement avec l'espace, gérer plus simplement les distances, simplifier l'organisation du parcours au cœur de la métapole. La configuration spatiale de Los Angeles est explicitement problématique pour tous les usagers de ces " transports en commun spécifiquement touristiques » : en l'absence de ces équipements, la manière des utilisateurs d'habiter touristiquement cette métapole ne serait sans doute pas aussi développée.

Il est possible, dans un premier temps, de comprendre ce système comme un dispositif permettant de ne pas traverser la métropole de façon indifférente, en allant strictement d'un point à un autre : les bus réalisent une boucle qui permet de l'investir au "ralenti ", de prendre le temps pour en découvrir certains lieux. Le cadre bâti n'est plus anonyme, indifférencié, mais acquiert une certaine épaisseur à travers une mise en récit mythologique : cela donne alors un autre sens à l'espace urbain et change le regard que les touristes peuvent en avoir. À Los Angeles le bus/van se substituerait en quelque sorte à la marche, métrique à laquelle les touristes ont classiquement recours pour s'approprier l'espace urbain des autres métropoles. Toujours est-il que cet aspect confirme et accentue, de façon non surprenante, l'importance de la métrique automobile dans la manière d'habiter touristiquement cette agglomération. L'espace urbain devient un 
paysage observé depuis des véhicules spécifiquement aménagés : on peut avancer l'idée qu'il s'agit là d'un véritable « drive-in tourist gaze ».

On constate, dans un deuxième temps, que cette manière de faire témoigne systématiquement de difficultés à faire avec cet espace de la part de ceux qui utilisent ces services, la décision d'y recourir s'apparentant souvent à un choix par défaut l'utilisation des vans est moins planifiée que subie pour la plupart des usagers: les touristes sont pris de court par la configuration spatiale de Los Angeles et la complexité à la maitriser afin de réaliser leurs pratiques. On peut avancer l'hypothèse que la pratique des circuits résulte de l'insuffisance de compétences de la part des individus pour visiter par eux-mêmes : ils font alors appel à des médiateurs pour filtrer et atténuer la difficulté des épreuves spatiales. Le bus touristique constitue une aide à la pratique qui intervient à au moins trois niveaux. Du point de vue des touristes, cet outil résout tout d'abord le problème du cheminement: une grande proportion des usagers ne sachant pas « où il faut aller ", le circuit leur donne accès à un parcours préétabli, ils n'ont pas besoin de sélectionner les lieux, les opérateurs réalisant cette tâche à leur place. Cela implique et renforce l'homogénéité des lieux pratiqués par les touristes - et c'est d'ailleurs une attente de la part d'une large majorité d'entre eux que de voir les lieux construits socialement comme étant ceux dignes d'intérêt. L'enjeu des métriques est également fondamental : le recours à ces services relève de la difficulté pour les touristes de gérer les distances par eux-mêmes. C'est enfin un outil pour gérer l'altérité: les touristes utilisent ces services pour faire l'expérience de lieux où ils n'oseraient pas aller par euxmêmes. Les individus compensent des compétences et des connaissances (qu'ils estiment) faibles par la mobilisation d'un capital économique en achetant une prestation pour ce qui correspond véritablement à une technologie spatiale au service de la pratique.

\section{BIBLIOGRAPHIE}

Jean-Pierre Augustin, « Los Angeles : la fabrication d'une mégapole de loisirs », Urbanisme, $\mathrm{n}^{\circ}$ 319, 2001.

Catherine cocKs, Doing the Town. The Rise of Urban Tourism in the United States 1859-1915, University of California Press, 2001.

Elisabeth CUNIN et Christian RINAUDO, «Visites guidées et marketing de la différence à Cartagena de Indias (Colombie) », Espaces et sociétés, vol. 135, n 4, 2008.

Margaret J. DANIELS, Laurlyn K. HARMON, Rodney VESE Jr., Minkyung PARK, Russell E. BRAYLEY "Spatial dynamics of tour bus transport within urban destinations », Tourism Management, vol. $64,2018$.

Philippe DUHAMEL et Rémy KNAFOU, « Le rôle du tourisme dans la construction et le fonctionnement de la centralité parisienne ", dans Thérèse SAINT-JULIEN et Renaud LE GOIX (dir.), La métropole parisienne. Centralités, inégalités, proximités, Belin, 2007.

Cynthia GHORRA-GOBIN, Los Angeles : le mythe américain inachevé, Éditions CNRS, 1997. 
Équipe MIT, Tourismes 1. Lieux communs, Belin, 2002.

Susan FAINSTEIN et David GLADSTONE, « Tourism in US Global Cities: A Comparison of New York and Los Angeles ", Journal of Urban Affairs, vol. 23, n 1, 2001.

Ignacio FARÍAS, « Spatial arrangements and mediation in sightseeing bus tours », dans Jana RICHTER (dir.), The Tourist City Berlin, Braun Publishing AG, 2010a.

Ignacio FARÍAS, « Sightseeing Buses: Cruising, Timing and the Montage of Attractions », Mobilities, vol. $5, \mathrm{n}^{\circ} 3,2010 \mathrm{~b}$.

Olivier LAZZAROTTI, Habiter : la condition géographique, Belin, 2006.

Jacques LÉvy, Le tournant géographique. Penser l'espace pour lire le monde, Belin, 1999.

Léopold LUCAS, Habiter touristique et agencement de l'espace urbain : le cas de Los Angeles. Recherche sur le concours des compétences des individus quant à leurs manières de faire avec les épreuves spatiales d'une métapole touristique, thèse de doctorat de l'Université de Lausanne, 2014.

Léopold LUCAS, « Les parcours des touristes à Los Angeles », Téoros, vol. 37, n 1, 2018 [http:// journals.openedition.org/teoros/3246].

Léopold LUCAS, « Los Angeles ou l'hypothèse de la métapole touristique », Mondes du Tourisme, numéro spécial « Tourisme et mondialisation », 2011.

Les M. LUMSDON, « Factors Affecting the design of tourism bus services », Annals of Tourism Research, vol. 33, n 3, 2006.

Michel LuSSAULT, L'avènement du monde. Essai sur l'habitation humaine de la Terre, Seuil, 2013.

Michel Lussault, De la lutte des classes à la lutte des places, Grasset, 2009.

Dean Maccannell, The Tourist. A New Theory of the Leisure Class, Schocken, 1976.

Hugo MARTIN, « Tensions are high as Hollywood tour bus operators vie for customers ", Los Angeles Times, 4 juillet 2015 [www.latimes.com/business/la-fi-hollywood-tourbus-wars-20150704story.html].

Hugo MARTIN, « As L.A. tourism rebounds, tour buses bring noise and gridlock », Los Angeles Times, 5 mars 2011 [http : //articles.latimes.com/2011/mar/05/business/la-fi-0305-tourbusnoise-20110305].

Jérôme MONNET, « Images de l'espace à Los Angeles : éléments de géographie cognitive et vernaculaire ", Culture et Ville, n 99, 1999.

Alfred schuTZ, Le chercheur et le quotidien, Méridiens Klincksieck, 1987.

Edward SoJA, « Le temps des nodalités post-métropolitaines », dans Sylvain ALLEMAND, François ASCHER et Jacques LÉVY (dir.), Les sens du mouvement, Belin, 2004.

Mathis STоск, «Faire avec de l'espace », dans Brigitte FRELAT-KAHN et Olivier LAZZAROTTI (dir.), Habiter vers un nouveau concept ?, Armand Colin, 2012.

Mathis Бтоск et Léopold LUCAS, « La double révolution urbaine du tourisme », Espaces et sociétés, vol. 3, n 151, 2012.

Richard WEINSTEIN, «The First American City », dans Allen scotT et Edward sojA (dir.), The City. Los Angeles and Urban Theory at the End of the $20^{\text {th }}$ Century, University of California Press, 1996.

Yves WINKIN, « Le touriste et son double », dans Yves WINKIN (dir.), Anthropologie de la communication, Seuil, 1997. 
Ludwig WITTGENSTEIN, Remarques philosophiques, Gallimard, 1984.

\section{NOTES}

1. Inversement, il ne faudrait pas non plus ignorer le fait que Los Angeles est une agglomération beaucoup plus urbaine que la plupart des autres métropoles américaines, avec un réseau de transports en commun parmi les plus développés et une densité parmi les plus importantes (voir Lucas, 2014). D'autre part, si l'on observe la dynamique urbaine, on constate depuis au moins une dizaine d'années une augmentation des métro-métriques (transports en communs) et une densification du Downtown, tout particulièrement à travers la dimension touristique, ce qui illustre la manière dont la sphère de la recréation participe d'un « retour du centre » tout autant que d'un « retour au centre » (voir Stock et Lucas, 2012).

2. Selon les chiffres qui m'ont été communiqués par Starline, la principale compagnie de circuits touristiques à Los Angeles, l'entreprise « carry over 500.000 passengers across over 20 tours. $80 \%$ are domestic and $20 \%$ international ».

3. «Brandycat » (25-34 ans, résidant dans le Berkshire), sur un forum de TripAdvisor datant du 7 février 2009: http : //www.tripadvisor.com/ShowTopic-g32655-i61-k2532197Open_top_bus_tour_of_LA-Los_Angeles_California.html

4. «Swdke» (homme, 35-49 ans, Ohio) post du 29 septembre 2008: www.tripadvisor.com/ ShowTopic-g32655-i61-k2281762-Starline_Movie_Stars_Homes_Tour-

Los_Angeles_California.html

5. «Lirwin13 ", (femme, 25-34 ans, Massachusetts), commentaire du 24 avril 2012 : www.tripadvisor.com/ShowTopic-g32655-i61-k5358338-Hollywood_Tour_of_the_stars_housesLos_Angeles_California.html

6. Cette photographie illustre d'autant plus cette pratique qu'elle a été prise par un touriste.

7. Une réponse aussi explicite que celle de Luis est assez rare, mais elle atteste que l'usage d'un guide ne va pas de soi, que tout le monde ne sait pas nécessairement utiliser cet outil de manière efficace.

8. De façon particulièrement illustrative, Scott (australien, la trentaine) développe une ruse par rapport à ces bus touristiques : « the first time, I followed the bus with my car. It was about 45 minutes. I heard everything they were saying about celebrities!». Parce que conduire à Los Angeles ne semble pas vraiment être un problème pour ce touriste, il a suivi l'un de ces véhicules et profité de la mise en discours sans payer le service. Cet épisode révèle explicitement le rôle des compétences au sein des manières de faire des individus.

\section{RÉSUMÉS}

Plutôt que d'envisager les bus touristiques dans la connotation péjorative d'une expression du tourisme « de masse », l'argument de cet article est de considérer ce service comme une véritable technologie spatiale qui aide les individus à gérer cet « horizon d'altérité » que constitue un lieu touristique. L'objectif est ainsi de souligner le fait que les individus ne sont pas tous capables, au même niveau, d'habiter touristiquement une métropole. Le point de départ consiste en effet à insister sur l'idée que «faire avec » de l'espace ne va pas de soi, n'est pas évident : habiter, c'est 
faire avec différentes épreuves spatiales. Pour gérer ces épreuves spatiales, l'individu doit mobiliser des compétences, définies ici comme la maîtrise de techniques. Dans cette perspective, l'hypothèse avancée dans cet article est que l'utilisation du bus touristique n'est pas seulement une pratique touristique en tant que telle, mais un outil utilisé par les individus quand ils estiment ne pas avoir la maîtrise suffisante de techniques pour découvrir par eux-mêmes une métropole. Cela est d'autant plus crucial à Los Angeles, l'étude de cas envisagée, où l'espace touristique s'étale sur une trentaine de kilomètres, augmentant le défi que constitue le parcours de la ville, où deux principaux types de services de transport collectif à vocation touristique différents coexistent.

Rather than considering tourist buses as a pejorative expression of mass tourism, this paper argues that it is a true spatial technology that helps individuals to manage the "otherness" of one tourist place. The purpose is to highlight the fact that individuals are not able, at the same level, to cope with space. Indeed, we insist on the idea that to "deal with" space is not obvious: we argue that to inhabit a place means to cope with some spatial stakes. To manage these epreuves, individual must mobilize skills, defined as the mastery of techniques. Then, we put forward the hypothesis that the use of tourist buses is not only a tourist practice as such, but a tool used by individuals when they feel they do not have sufficient mastery of techniques to discover by themselves a metropolis. This is crucial in Los Angeles, our case study, where the tourist space is spread over thirty kilometers, increasing the challenge of moving within the city, and where two main types of tourist buses coexist.

\section{INDEX}

Mots-clés : compétences, distances, épreuves spatiales, pratiques, Los Angeles

Keywords : skills, distances, spatial stakes, practice, Los Angeles

\section{AUTEUR \\ LÉOPOLD LUCAS}

Géographe

PhD., Maître-assistant

Institut Géographie et Durabilité

Université de Lausanne

leopold.lucas@unil.ch 